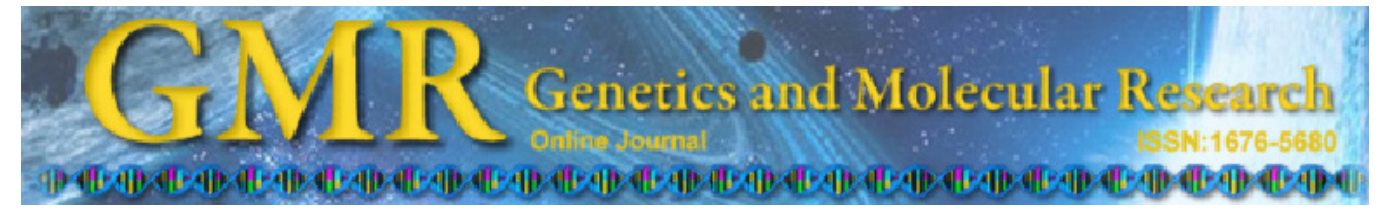

\title{
Effect of atorvastatin on left atrial function of patients with paroxysmal atrial fibrillation
}

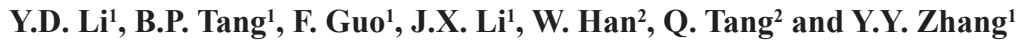 \\ ${ }^{1}$ Pacing and Electrophysiology Department, \\ The First Affiliated Hospital of Xinjiang Medical University, \\ Urumqi, Xinjiang, China \\ ${ }^{2}$ Cardiac Ultrasound Department, \\ The First Affiliated Hospital of Xinjiang Medical University, \\ Urumqi, Xinjiang, China \\ Corresponding author: B.P. Tang \\ E-mail: baopengtang123@163.com
}

Genet. Mol. Res. 12 (3): 3488-3494 (2013)

Received February 13, 2013

Accepted August 2, 2013

Published September 10, 2013

DOI http://dx.doi.org/10.4238/2013.September.10.5

\begin{abstract}
The aim of this study was to evaluate the effects of atorvastatin on left atrial (LA) function in paroxysmal atrial fibrillation patients. Fifty-eight paroxysmal atrial fibrillation patients were divided into two groups (treatment and control groups). The echocardiography parameters, including LA active emptying volume (LAAEV), LA active emptying fraction, LA maximum volume, LA total emptying volume, LA total emptying fraction, and LA ejection force (LAEF), were measured before treatment, and then 12 and 18 months after treatment. Compared to pre-treatment levels, the parameters reflecting LA pump function, such as LAAEV and LAEF, decreased significantly in treatment groups 12 months after treatment $(\mathrm{P}<0.05)$. LAAEV and LAEF significantly increased 18 months after treatment $(\mathrm{P}<0.05)$, and the indicators reflecting LA reservoir function, such as maximum volume, total emptying volume, and total emptying fraction increased significantly 18 months after treatment $(\mathrm{P}<0.05)$. Compared with pre-treatment levels, LAAEV and LAEF decreased significantly 18 months after treatment in the control group ( $\mathrm{P}$ $<0.05)$. These results demonstrated that long-term atorvastatin treatment
\end{abstract}


could ameliorate the function of the atrium sinistrum.

Key words: Cardiology; Atrial fibrillation; Echocardiography; Atorvastatin; Left atrial function

\section{INTRODUCTION}

Atrial fibrillation (AF) is one of the most common arrhythmias observed in clinics. The onset of AF is closely associated with age. Although AF can occur at any age, the incidence of the disease in children is rare, and is more common in older people. In the general population, its incidence reaches $0.4 \%$. With increasing age, the incidence rate of AF is significantly increased, reaching $6 \%$ in patients older than 65 years old, $10 \%$ in patients greater than 75 years old, and $20 \%$ in patients 85 years old or older (Nattel et al., 2008). The possible reasons and/or mechanisms causing AF include electrophysiological abnormalities, an increase in atrial pressure, cardiac atrium ischemia, surgery, inflammation or infiltrative atrial disease, and drugs and endocrine diseases, among others. Although the exact mechanisms of the preventative effect of statins on AF recurrence remain unclear, it may be related to their anti-inflammatory (Dernellis and Panaretou, 2004; Lob et al., 2005) and antioxidant effects, which inhibit the progress of coronary artery disease. In addition, it may be related to anti-arrhythmic effects caused by changes in ion channels (Fuster et al., 2006). The incidence and maintenance of AF is related to electrical remodeling and tissue remodeling of the cardiac atrium, and is related to the activation of the atrial tissue angiotensin system, which is one of the pathophysiological foundations contributing to sustained AF and its development into a chronic process. Therefore, interventions of the pathophysiological process may help to reduce the incidence of AF (Healey et al., 2005).

Circumferential pulmonary vein electrical isolation vestibular surgery, as a main AF catheter ablation surgical method, can destroy the trigger of AF and maintain the matrix, and is therefore one of the most effective methods used for treating AF in recent years (Pappone et al., 2000). However, recurrence rates following surgery remain high (Pappone et al., 2001; Oral et al., 2002; Arentz et al., 2003), which combined with the high cost of surgery, limit its clinical applications. Currently, drug treatment is the main therapeutic tool for AF. Statins have several broad effects independent of their lipid modulation functions. Recent studies have shown that statins can inhibit the electrical and structural remodeling associated with AF. The present study was designed to detect indicators reflecting left atrial systolic and diastolic function by ultrasound, and to investigate the short-term impact of atorvastatin on left atrial (LA) function in paroxysmal AF (PAF) patients.

\section{MATERIAL AND METHODS}

\section{Subjects}

Fifty-eight cases of patients with PAF were selected in this study in hospital from January 2006 to June 2008. Their diagnoses were in line with the 2006 ACC/AHA/ESC treatment guidelines for AF classification, and the inclusion criteria and general situation of patients were as follows: i) the electrocardiogram (ECG) and Holter results showed that AF occurred at least twice, and was transferred into sinus rhythm within 7 days; the mean duration of AF was $34.2 \pm$ $32.5 \mathrm{~h} /$ episode, and the mean frequency of episodes was $10.9 \pm 5.9$ times/week; ii) therapy with 
one or more anti-arrhythmic drugs was ineffective or difficult to tolerate for patients; iii) no significant LA enlargement ( $\leq 45 \mathrm{~mm}$ in diameter) and thrombus on LA appendage were ruled out by esophagus ultrasound; iv) patients with valvular heart disease, dilated cardiomyopathy, severe left ventricular dysfunction, and other organic heart diseases were excluded, and patients with PAF induced by hypertension, hyperthyroidism, or alcohol were also excluded; v) 43 cases were males, 15 were females, aged $51.5 \pm 11.5$ years. The mean history of AF patients was 7.2 \pm 46.4 months.

\section{Experimental design}

The patients were randomly divided into two groups: the treatment group $(\mathrm{N}=30$, average age: $58.5 \pm 15.2$ years), and the control group $(\mathrm{N}=28$ patients, average age $58.2 \pm 13.8$ years). There were no statistically significant differences between the two groups in age, weight, blood pressure, lipid levels, family history of coronary heart disease, diabetes mellitus and smoking history, LA diameter, P-wave maximum time limit, and P-wave dispersion, or other general patient indicators. The 28 patients in the control group were treated with oral amiodarone (Hangzhou Sanofi-Synthelabo Minsheng Pharmaceutical Co., Ltd., China); $200 \mathrm{mg}$ three times per day the first week, $200 \mathrm{mg}$ twice a day the second week, and $200 \mathrm{mg}$ once a day the third week. Patients in the treatment group were treated with $20 \mathrm{mg}$ atorvastatin (Pfizer, USA) per day for 18 months, taken once after dinner every night. No patient in either group was treated with angiotensin-converting enzyme inhibitor or angiotensin II receptor blocker drugs.

\section{Evaluation of LA function and index calculations}

Cardiac ultrasounds were performed on all patients both before and after treatment when their heart rates were in sinus rhythm. The cardiac ultrasound instrument used was the GE VIVID 7 type, and the frequency of the probe was set to 1.7-3.4 MHz. The patients were placed in the left lateral position, asked to breathe calmly, and the following indices were measured: 1) with the guide of the M-mode ultrasound, the LA anteroposterior diameter of the patients was measured on the parasternal long axis of left ventricle view at the end of contraction, 2) upon opening or closure of the mitral valve, and at the beginning of atrial contraction (start of the P-wave in the ECG), the LA maximum volume (LAVmax), LA minimal volume (LAVmin), and LA volume P-wave (LAVP) of patients were measured, 3) the two-dimensional four-chamber of the cardiac apex and the two-chamber dynamic image were retained, and the biplane Simpson method was used to measure the volumes on the endsystole and end-diastole to calculate the left ventricular ejection fraction. Heart rates of the patients were also detected. All of these indices were measured over three consecutive cardiac cycles. The LA active emptying volume (LAAEV) was calculated based on the following formula: LAAEV $=$ LAVP - LAVmin. The LA active emptying fraction (LAAEF) was calculated as: $\mathrm{LAAEF}=\mathrm{LAAEV} / \mathrm{LAVP}$. The LA total emptying volume (LATEV) was calculated as: LATEV = LAVmax - LAVmin. The LA total emptying fraction (LATEF) was calculated as: LATEF $=$ LATEV $/$ LAVmax, and the LA ejection force $(\mathrm{LAEF})=(1 / 2) \times \rho \times(\mathrm{MVA}) \times(\mathrm{VA} 2)$, where $\rho$ is the blood density $\left(1.06 \mathrm{~g} / \mathrm{cm}^{3}\right)$.

\section{Follow-up of patients after treatment}

All patients were followed up for 12 and 18 months after treatment. Assessments in- 
cluded clinical symptoms, 12-lead ECG, 24-h Holter monitoring, and the aforementioned relevant echocardiography indicators. The echocardiography measurements of each patient were determined by two professional sonographers. The average of the data from the two operators was used for the final analysis.

\section{Statistical analysis}

The data were analyzed with the SPSS 13.0 statistical software, and measurement data are reported as means \pm standard deviation. Means between groups were compared with analysis of variance (ANOVA). The independent sample test was adopted for comparisons before and after treatment. $\mathrm{P}<0.05$ was considered to be statistically significant.

\section{RESULTS}

\section{Condition of patients after treatment}

All 58 patients were successfully followed-up to completion after treatment.

\section{Changes in LA function before and after treatment}

The heart rates and LVEF of patients did not change significantly after treatment (Table 1). The indices reflecting LA auxiliary pump function, including LAAEV, LAAEF, and LAEF, did not change significantly in patients in the treatment group 12 months after treatment compared to pre-treatment levels $(\mathrm{P}>0.05)$. However, these indices significantly increased 18 months after treatment compared to pre-treatment levels $(\mathrm{P}<0.05)$ (Table 2$)$. The indices reflecting LA storage function, including LAVmax, LATEV, and LATEF, of patients in the treatment group did not significantly change 12 months after treatment $(\mathrm{P}>0.05)$, whereas they significantly increased 18 months after treatment compared to pre-treatment levels $(\mathrm{P}<0.05)$ (Table 3$)$.

Table 1. Changes of heart rates (HR) and left ventricular ejection fraction (LVEF) of the patients on the treatment group $(\mathrm{N}=30)$ and the control group $(\mathrm{N}=28)$ before and after treatment.

\begin{tabular}{lccccc}
\hline Time & \multicolumn{2}{c}{ HR $(\mathrm{bpm})$} & & \multicolumn{2}{c}{ LVEF (\%) } \\
\cline { 2 - 3 } & Treatment group & Control group & & Treatment group & Control group \\
\hline Before treatment & $67.5 \pm 6.5$ & $69.5 \pm 6.2$ & $51 \pm 3$ & $52 \pm 2$ \\
12 months after treatment & $68.5 \pm 6.4$ & $65.5 \pm 6.4$ & $68.5 \pm 6.5$ & $54 \pm 4$ & $53 \pm 2$ \\
18 months after treatment & $68.5 \pm 6.6$ & & $54 \pm 3$ & $56 \pm 3$ \\
\hline
\end{tabular}

\begin{tabular}{|c|c|c|c|c|c|}
\hline Time & $\operatorname{LAVP}\left(\mathrm{cm}^{3}\right)$ & LAAPD $(\mathrm{cm})$ & LAAEV $\left(\mathrm{cm}^{3}\right)$ & LAAEF & LAEF (dynes) \\
\hline Before treatment & $25.2 \pm 2.5$ & $40.3 \pm 4.5$ & $7.5 \pm 2.8$ & $0.45 \pm 0.2$ & $15.6 \pm 3.5$ \\
\hline 12 months after treatment & $25.7 \pm 2.3$ & $39.4 \pm 4.0^{\#}$ & $5.6 \pm 3.1$ & $0.33 \pm 0.2$ & $13.4 \pm 4.0$ \\
\hline 18 months after treatment & $25.8 \pm 2.4$ & $37.4 \pm 3.5^{\#}$ & $8.2 \pm 2.9^{\#}$ & $0.46 \pm 0.3^{\#}$ & $16.7 \pm 3.9^{\#}$ \\
\hline
\end{tabular}


Table 3. Changes of the indexes reflecting storage function of the patients on the treatment group before and after treatment $(\mathrm{N}=30)$.

\begin{tabular}{|c|c|c|c|c|c|}
\hline Time & $\mathrm{VA}(\mathrm{cm} / \mathrm{s})$ & A-VTI (cm) & LAVmax $\left(\mathrm{cm}^{3}\right)$ & LATEV $\left(\mathrm{cm}^{3}\right)$ & LATEF $(\%)$ \\
\hline Before treatment & $61.0 \pm 11.2$ & $4.3 \pm 1.3$ & $32.6 \pm 4.1$ & $14.0 \pm 3.2$ & $55.0 \pm 19$ \\
\hline 12 months after treatment & $51.4 \pm 10.3$ & $3.5 \pm 1.2$ & $33.5 \pm 4.4$ & $13.0 \pm 3.5$ & $56.0 \pm 18$ \\
\hline 18 months after treatment & $64.3 \pm 11.2^{\#}$ & $4.8 \pm 1.4^{\#}$ & $38.1 \pm 4.5^{\#}$ & $16.0 \pm 3.7^{\#}$ & $59.0 \pm 20^{\#}$ \\
\hline
\end{tabular}

${ }^{\#} \mathrm{P}<0.05$, compared with before treatment. $\mathrm{VA}=$ peak velocity of A wave in late diastole; A-VTI = velocity-time integral of A wave; LAVmax = left atrium (LA) maximum volume; LATEV = LA total emptying volume; LATEF $=$ LA total emptying fraction.

The relapse frequency of patients in the control group was $5.5 \pm 2.5$ times/week, and the duration of the episodes was $21.5 \pm 17.5 \mathrm{~h}$. The indices reflecting LA auxiliary pump function, including LAAEV, LAAEF, and LAEF, of the control group were significantly reduced after treatment compared with pre-treatment levels $(\mathrm{P}<0.05)$ (Table 4). However, the indices reflecting LA storage function, including LAVmax, LATEV, and LATEF did not change significantly after treatment in the control group $(\mathrm{P}>0.05)$ (Table 5).

Table 4. Changes of the indexes reflecting left atrial (LA) auxiliary pump function before and after treatment on the control group $(\mathrm{N}=28)$.

\begin{tabular}{|c|c|c|c|c|c|}
\hline Time & $\operatorname{LAVP}\left(\mathrm{cm}^{3}\right)$ & LAAPD $(\mathrm{cm})$ & $\operatorname{LAAEV}\left(\mathrm{cm}^{3}\right)$ & LAAEF & LAEF (dynes) \\
\hline Before treatment & $26.6 \pm 2.6$ & $41.5 \pm 4.3$ & $7.4 \pm 2.5$ & $0.38 \pm 0.1$ & $14.5 \pm 3.3$ \\
\hline 12 months after treatment & $26.8 \pm 2.3$ & $41.4 \pm 4.0$ & $5.5 \pm 3.0^{\#}$ & $0.28 \pm 0.3^{\#}$ & $12.5 \pm 3.0^{\#}$ \\
\hline 18 months after treatment & $26.6 \pm 2.6$ & $39.4 \pm 3.5$ & $5.0 \pm 2.9^{\#}$ & $0.26 \pm 0.4^{\#}$ & $11.4 \pm 3.7^{\#}$ \\
\hline
\end{tabular}

${ }^{\#} \mathrm{P}<0.05$, compared with before treatment. For abbreviations, see legend to Table 2.

Table 5. Changes of the indexes reflecting storage function of the patients on the control group before and after treatment $(\mathrm{N}=28)$.

\begin{tabular}{|c|c|c|c|c|c|}
\hline Time & $\mathrm{VA}(\mathrm{cm} / \mathrm{s})$ & A-VTI $(\mathrm{cm})$ & LAVmax $\left(\mathrm{cm}^{3}\right)$ & $\operatorname{LATEV}\left(\mathrm{cm}^{3}\right)$ & LATEF (\%) \\
\hline Before treatment & $59.0 \pm 11.2$ & $4.1 \pm 1.1$ & $34.9 \pm 4.8$ & $14.2 \pm 4.2$ & $52 \pm 18$ \\
\hline 12 months after treatment & $51.5 \pm 9.5^{\#}$ & $3.3 \pm 1.3^{\#}$ & $32.5 \pm 4.6$ & $12.7 \pm 3.5$ & $53 \pm 15$ \\
\hline 18 months after treatment & $50.0 \pm 9.2^{\#}$ & $3.2 \pm 1.3^{\#}$ & $30.4 \pm 4.1$ & $12.5 \pm 3.7$ & $51 \pm 20$ \\
\hline
\end{tabular}

${ }^{\#} \mathrm{P}<0.05$, compared with before treatment. For abbreviations, see legend to Table 3.

\section{DISCUSSION}

Atorvastatin is a widely used lipid-lowering statin drug in clinical settings. Statins can inhibit the rate-limiting enzyme HMG-coenzyme A reductase at early stages of cholesterol synthesis in liver cells to reduce blood cholesterol levels of patients. Statins also improve endothelial function, stabilize atherosclerotic plaque, inhibit proliferation of vascular smooth muscle cells, reduce vascular inflammatory reactions, and in particular, have anti-inflammatory effects independent of lipid-lowering effects. Statins can inhibit expressions of cell adhesion molecules and tissue factors, prevent monocyte adhesion binding to vascular endothelium and entering the subendothelial space, avoid formation of foam cells and release of inflammatory cytokines, reduce C-reactive protein levels (Weber et al., 1997; Strandberg et al., 1999), and inhibit degeneration and fibrosis of myocardial cells. Luo et al. (1999) found that simvastatin could reduce the activity of the angiotensin-converting enzyme by $36 \%$, and decreased the production of angiotensin II by $11 \%$, which changed atrial structural and electrical remodel- 
ing, and reduced the incidence of AF. Previous studies have found that after patients with AF were treated with drugs or electrical cardioversion and sinus rhythm was recovered, LA function also gradually recovered (Beukema et al., 2005; Takahashi et al., 2007). Ozaydin et al. (2006) performed a prospective and randomized study to evaluate the effects of atorvastatin in patients with $\mathrm{AF}$ recurrence after electrical cardioversion. Cox proportional model analysis revealed the following risk factors as predictors of recurrent AF: body mass index 25-30 and $\geq 30 \mathrm{~kg} / \mathrm{m}^{2}, \mathrm{AF} \geq 3$ months, diabetes, and LA $\geq 45 \mathrm{~mm}$ in diameter. After the predictors were corrected, the reduction in recurrence risk of the AF group by atorvastatin remained significant (Ozaydin et al., 2006). The present study demonstrated that the LA anteroposterior diameter of patients in the treatment group decreased 12 months after treatment, and the indices reflecting the auxiliary pump and storage function of atrium sinistrum were decreased, which suggested that left atrial structural reverse remodeling occurred in a relatively short period of time when sinus rhythms of the patients were restored after treatment, and recovery of LA function was slower than atrial structural inverse remodeling. This level was reduced below pre-treatment levels, which indicated that the function of atrial muscle cells cannot be fully restored to physiological state shortly after AF becomes sinus rhythm, and is still at stunning state to affect electrical-mechanical coupling process of cardiac cells.

Eighteen months after treatment, the indices reflecting LA auxiliary pumps and storage function increased significantly, which suggested that with long-term maintenance of sinus rhythm, LA function was subsequently improved. Thomas et al. (1998) showed that the material basis of AF electrical remodeling was abnormal changes at the cellular and molecular level. Animal hearts with AF often show abnormal synthesis of inter-cellular adhesion molecules and other cells, increased synthesis of $\mathrm{Cx} 40$ and $\mathrm{Cx} 43$, which are related to increasing anisotropy in atrial tissue, increasing conduction speed, and the formation of local microreentry. Possibly due to the reversal of electrical remodeling over a long period of treatment, the abnormal synthesis of cell adhesion molecules connected to other cells was significantly reduced, which significantly improved LA function.

Abuissa et al. (2009) suggested that statins could improve the relapse rate and prognosis of AF patients. Mariscalco et al. (2007) conducted an observational and retrospective study to evaluate the effect of statin treatment before coronary artery bypass graft surgery, in order to reduce the incidence of post-operative AF. Pre-treatment of $40 \mathrm{mg} /$ day atorvastatin reduced the incidence of new-onset AF in elective cardiac surgery patients, and shortened the hospital stay of these patients. In this experiment, the joint study of amiodarone combined with atorvastatin in the prevention of the recurrence showed that the treatment significantly reduced AF occurrence in PAF patients, and the effect of the maintenance of sinus rhythm was superior to that of amiodarone, which was consistent with results of Henningsen et al. (2009) and Schnabel et al. (2010), who showed many of the mapped atrial fibrillation recurrence rate and prognosis. Together, these results provide a more convenient and feasible way to predict the prognosis of patients with AF in future clinical research.

\section{REFERENCES}

Abuissa H, O'Keefe JH and Bybee KA (2009). Statins as antiarrhythmics: a systematic review part I: effects on risk of atrial fibrillation. Clin. Cardiol. 32: 544-548.

Arentz T, Von Rosenthal J, Blum T, Stockinger J, et al. (2003). Feasibility and safety of pulmonary vein isolation using a new mapping and navigation system in patients with refractory atrial fibrillation. Circulation 108: 2484-2490. 
Beukema WP, Elvan A, Sie HT, Misier AR, et al. (2005). Successful radiofrequency ablation in patients with previous atrial fibrillation results in a significant decrease in left atrial size. Circulation 112: 2089-2095.

Dernellis J and Panaretou M (2004). Relationship between C-reactive protein concentrations during glucocorticoid therapy and recurrent atrial fibrillation. Eur. Heart J. 25: 1100-1107.

Fuster V, Ryden LE, Cannom DS, Crijns HJ, et al. (2006). ACC/AHA/ESC 2006 Guidelines for the management of patients with atrial fibrillation: a report of the American College of Cardiology/American Heart Association Task Force on Practice Guidelines and the European Society of Cardiology Committee for Practice Guidelines (Writing Committee to Revise the 2001 Guidelines for the Management of Patients With Atrial Fibrillation): developed in collaboration with the European Heart Rhythm Association and the Heart Rhythm Society. Circulation 114: e257-e354.

Healey JS, Morillo CA and Connolly SJ (2005). Role of the renin-angiotensin-aldosterone system in atrial fibrillation and cardiac remodeling. Curr. Opin. Cardiol. 20: 31-37.

Henningsen KM, Therkelsen SK, Bruunsgaard H, Krabbe KS, et al. (2009). Prognostic impact of hs-CRP and IL-6 in patients with persistent atrial fibrillation treated with electrical cardioversion. Scand. J. Clin. Lab. Invest. 69: 425-432.

Lo B, Fijnheer R, Nierich AP, Bruins P, et al. (2005). C-reactive protein is a risk indicator for atrial fibrillation after myocardial revascularization. Ann. Thorac. Surg. 79: 1530-1535.

Luo JD, Zhang WW, Zhang GP, Guan JX, et al. (1999). Simvastatin inhibits cardiac hypertrophy and angiotensinconverting enzyme activity in rats with aortic stenosis. Clin. Exp. Pharmacol. Physiol. 26: 903-908.

Mariscalco G, Lorusso R, Klersy C, Ferrarese S, et al. (2007). Observational study on the beneficial effect of preoperative statins in reducing atrial fibrillation after coronary surgery. Ann. Thorac. Surg. 84: 1158-1164.

Nattel S, Burstein B and Dobrev D (2008). Atrial remodeling and atrial fibrillation: mechanisms and implications. Circ. Arrhythm. Electrophysiol. 1: 62-73.

Oral H, Knight BP, Tada H, Ozaydin M, et al. (2002). Pulmonary vein isolation for paroxysmal and persistent atrial fibrillation. Circulation 105: 1077-1081.

Ozaydin M, Varol E, Aslan SM, Kucuktepe Z, et al. (2006). Effect of atorvastatin on the recurrence rates of atrial fibrillation after electrical cardioversion. Am. J. Cardiol. 97: 1490-1493.

Pappone C, Rosanio S, Oreto G, Tocchi M, et al. (2000). Circumferential radiofrequency ablation of pulmonary vein ostia: A new anatomic approach for curing atrial fibrillation. Circulation 102: 2619-2628.

Pappone C, Oreto G, Rosanio S, Vicedomini G, et al. (2001). Atrial electroanatomic remodeling after circumferential radiofrequency pulmonary vein ablation: efficacy of an anatomic approach in a large cohort of patients with atrial fibrillation. Circulation 104: 2539-2544.

Schnabel RB, Larson MG, Yamamoto JF, Sullivan LM, et al. (2010). Relations of biomarkers of distinct pathophysiological pathways and atrial fibrillation incidence in the community. Circulation 121: 200-207.

Strandberg TE, Vanhanen H and Tikkanen MJ (1999). Effect of statins on C-reactive protein in patients with coronary artery disease. Lancet 353: 118-119.

Takahashi Y, O’Neill MD, Hocini M, Reant P, et al. (2007). Effects of stepwise ablation of chronic atrial fibrillation on atrial electrical and mechanical properties. J. Am. Coll. Cardiol. 49: 1306-1314.

Thomas SA, Schuessler RB, Berul CI, Beardslee MA, et al. (1998). Disparate effects of deficient expression of connexin43 on atrial and ventricular conduction: evidence for chamber-specific molecular determinants of conduction. Circulation 97: 686-691.

Weber C, Erl W, Weber KS and Weber PC (1997). HMG-CoA reductase inhibitors decrease CD11b expression and CD11b-dependent adhesion of monocytes to endothelium and reduce increased adhesiveness of monocytes isolated from patients with hypercholesterolemia. J. Am. Coll. Cardiol. 30: 1212-1217. 